\title{
Analyzing Modulation Techniques for the Modular Multilevel Converter
}

\author{
Ngoc-ThinhQuach ${ }^{1}$, Sang Heon Chae ${ }^{1}$, Seungmin Lee ${ }^{2}$,Ho-Chan Kim¹, Eel-Hwan Kim* \\ ${ }^{1}$ Department of Electrical Engineering, Jeju National University, South Korea. \\ ${ }^{2}$ Faculty of Wind Energy Engineering Graduate School, Jeju National University, South Korea. \\ * Corresponding author. Email: ehkim@jejunu.ac.kr \\ Manuscript submitted February 15, 2016; accepted August 2, 2016. \\ doi: 10.17706/ijcee.2016.8.4.259-271
}

\begin{abstract}
Modular multilevel converter is one of the new technologies that are developed for the multilevel converter topology. It has some advantages over the conventional converters such as high capacity, high efficiency and low total harmonic distortion. The harmonic concept relates closely to number of levels of output voltage and modulation techniques. Thus, this paper presents an analysis of different modulation techniques, namely sinusoidal pulse width modulation, third harmonic injection pulse width modulation and nearest level modulation, for the modular multilevel converter. The objective is to find out a suitable modulation technique that can reduce the total harmonic distortion and improve the efficiency of modular multilevel converter. The simulation results are carried out by using PSCAD/EMTDC simulation program.
\end{abstract}

Key words: Modular multilevel converter, modulation technique, pulse width modulation, third harmonic injection, nearest level modulation.

\section{Introduction}

The concept of the multilevel converter has been known well in the past such as diode-clamped multilevel converter [1], flying-capacitors multilevel converter [2] and cascade multilevel converter [3]. Recently, a new topology of the multilevel converter has been developed which is called by a modular multilevel converter (MMC) [4]. The MMC is a type of voltage source converter (VSC). However, it has many advantages over other VSCs such as low harmonic, high efficiency, high capacity and so on [5]-[7]. Thus, the MMC is a promising converter topology in the near future. The control objective of the MMC is similar to the conventional converters [8]. However, there are some difference points. One of them is modulation technique because the MMC is structured by a specific number of submodules (SMs).Generally, the modulation techniques of the MMC may be classified into selective harmonic elimination pulse width modulation (SHEPWM), optimized harmonic stepped waveform (OHSW), sinusoidal pulse width modulation (SPWM), third harmonic injection pulse width modulation (THIPWM), space vector modulation (SVM) and nearest level modulation (NLM) [9]-[13]. Each technique has its own advantages and disadvantages. The SHEPWM and OHSW techniques are very complicated because the solution of a large number of equations to get optimal switching angles [9]. The SVM technique is widely used. However, the application of the SVM for a MMC is also complicated because the difficulty in determining the location of the reference vector, the calculation of on-times, and determination and selection of switching states [12]. Among modulation techniques, the SPWM and THIPWM are simple. A modulating signal is compared to the multicarrier waveforms to generate the gating signals for the power semiconductor devices [10], [11]. In 
[10], the authors mentioned three kinds of multicarrier waveforms such as phase disposition (PD), phase opposite disposition (POD) and alternative phase opposite disposition (APOD). In [11], only two kinds of multicarrier waveforms were proposed such as phase shifted (PS) and PD. Normally, the SPWM and THIPWM will cause high power loss because of high switching frequency. To improve this problem, the NLM has been used [13]. The power semiconductor devices are only switched on/off once every level. Therefore, the switching frequency and power loss are reduced significantly.

It is accepted that the performance of a converter with any modulation techniques can relate to the harmonic contents of the output voltage and switching losses. However, there is no a comprehensive analysis of modulation techniques for the MMC yet. Thus, this paper presents an analysis of different modulation techniques for the MMC. The objective is to find out a suitable modulation technique that can reduce the total harmonic distortion and improve the efficiency for the MMC. Three modulation techniques will be considered, namely SPWM, THIPWM and NLM.

The rest of this paper is organized as follows. Section 2 explains briefly the structure and operating principle of the MMC. The modulation techniques are analyzed in Section 3. Section 4 is the simulation results and discussion. Section 5 draws the conclusion.

\section{Modular Multilevel Converter}

The basic structure of the MMC is shown in Fig. 1. It is structured by six arms. Each arm has a total of $N$ SMs connected in series and a series inductor which provides a current control within the phase arms and limits fault currents. Two arms in the same leg comprise a phase unit. A SM is a half-bridge cell that consists of two IGBTs, two anti-parallel diodes and a capacitor. The AC side of the MMC is connected to the unity grid through a Y- $\Delta$ three-phase transformer.

Each arm of the MMC can operate as a controllable voltage source. Six arms are corresponding to the six controllable voltage sources. These sources must meet the criterion that their voltage sum always has to match the constant DC-link voltage. Thus, if the magnitude of the voltage source in the upper arm is increased, the magnitude of the voltage source in the lower arm must be decreased and vice versa. In the $\mathrm{MMC}$, the maximum number of levels of the AC output voltage is related strictly to the number of SMs per each arm. If there are $N$ SMs per each arm, the number of levels of the output voltage will be $N+1$. Each level will be generated depending on the number of SMs in the on-state in upper and lower arms. At any time, $N$ SMs have to be in the on-state and $N$ SMs have to be in the off-state in each phase. This ensures that the DC-link voltage always has a constant value. The value of each level is $V_{d c} / N$. The output voltage waveform of the MMC is shown in Fig. 2.

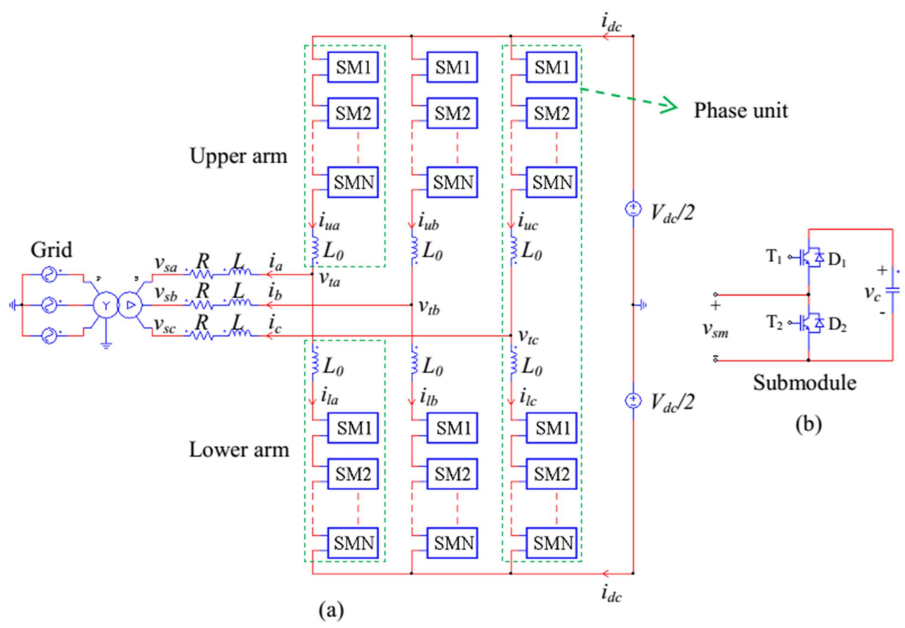

Fig. 1. Circuit diagram of the MMC. 


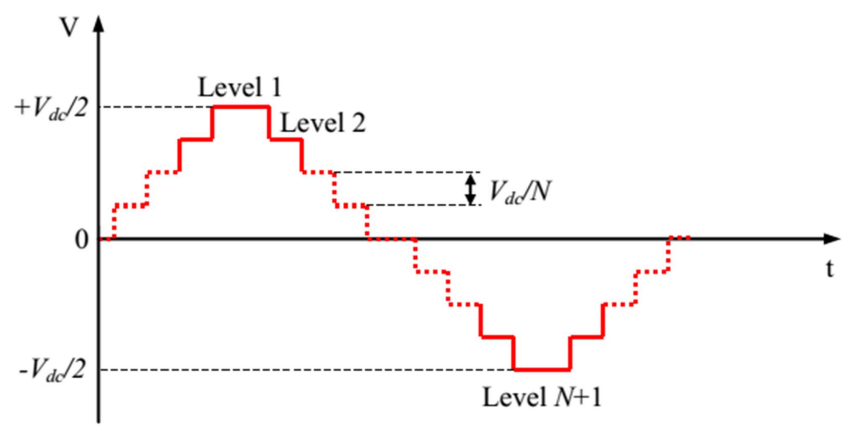

Fig. 2. Output voltage waveform of the MMC.

\section{Modulation Technique}

The modulation techniques of the MMC may be classified into SHEPWM, OHSW, SPWM, THIPWM, SVM and NLM. In this paper, the principle of the SPWM, THIPWM and NLM which are most commonly used for the MMC are analyzed and compared to each other. With the MMC, the PWM technique is created by a modulating signal and several carrier waveforms. Because of using many carrier waveforms, the PWM technique for the MMC is considered as a multicarrier PWM technique. The modulating signal may be a sinusoidal PWM or a third harmonic injection PWM. To illustrate for the modulation techniques, a 11-level MMC is taken as an example.

\subsection{Multicarrier PWM Technique}

Depending on the arrangement of the carrier waveforms, the multicarrier PWM technique is classified into categories as follows.

\subsubsection{Phase shifted pulse width modulation}

A MMC with $(N+1)$ levels requires $N$ triangular carrier waveforms. In the phase-shifted PWM (PS-PWM) technique, all carrier waveforms have the same frequency and the same peak-to-peak amplitude, but there is a phase shift between two adjacent carrier waveforms. This phase shift is given by

$$
\varphi_{c r}=\frac{360^{\circ}}{N}
$$

The arrangement of the carrier waveforms is show in Fig. 3. In this technique, the harmonics are concentrated as sidebands around $N . f_{c r}$. The signal, that decides the switching frequency, is equal to the carrier frequency.

\subsubsection{Level shifted pulse width modulation}

The level shifted PWM (LS-PWM) technique also requires $N$ carrier waveforms. However, these carrier waveforms are vertically disposed and their bands are contiguous. Each carrier waveform only covers a small portion of the modulating signal range, and it can affect at a time on the output signal. Total switching of all SMs in each arm will be equal to the carrier frequency. The harmonics are presented as sidebands around the carrier frequency, $f_{c r}$. This is also one of the different points to the PS-PWM technique.

Depending on the phase disposition of the carrier waveforms, the LS-PWM technique can be classified into three cases such as PD, POD and APOD.

\subsubsection{Phase disposition pulse width modulation}

In the PD technique, all carrier waveforms are in phase. Each carrier waveform is different to the adjacent one by the voltage offset as shown in Fig. 4. 


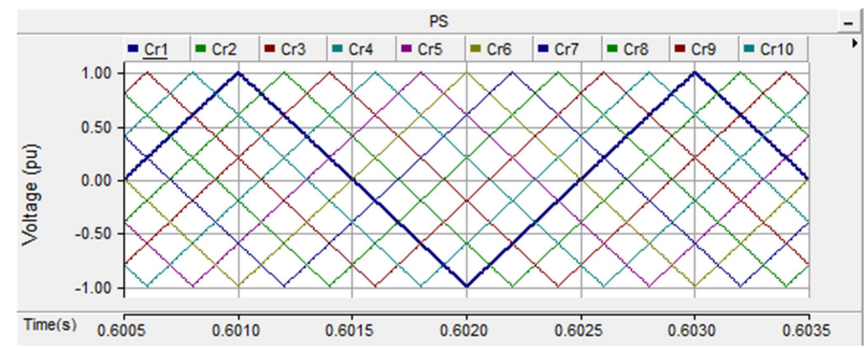

Fig. 3. Phase shifted PWM technique.

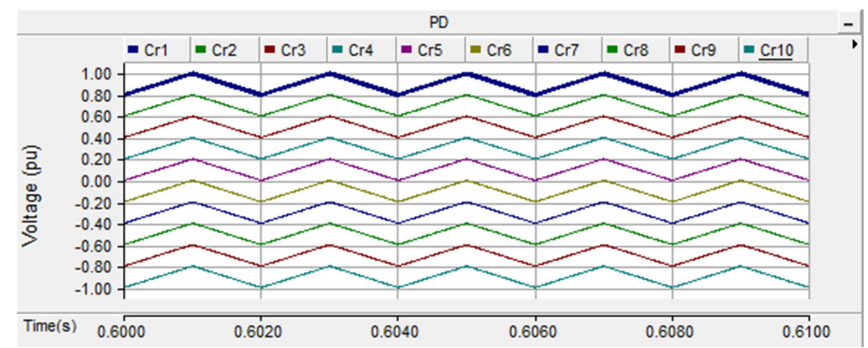

Fig. 4. Phase disposition PWM technique.

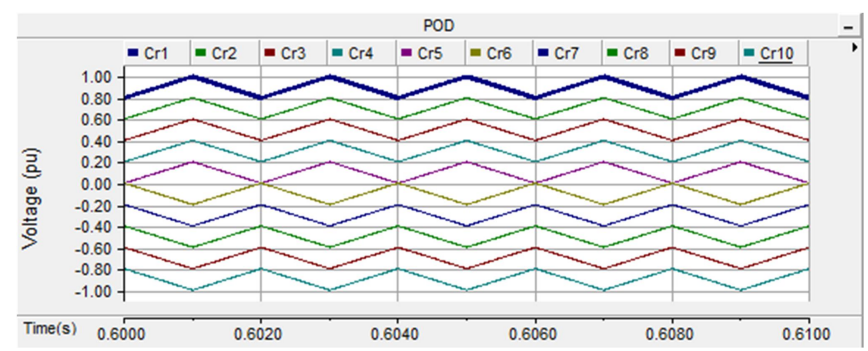

Fig. 5. Phase opposite disposition PWM technique.

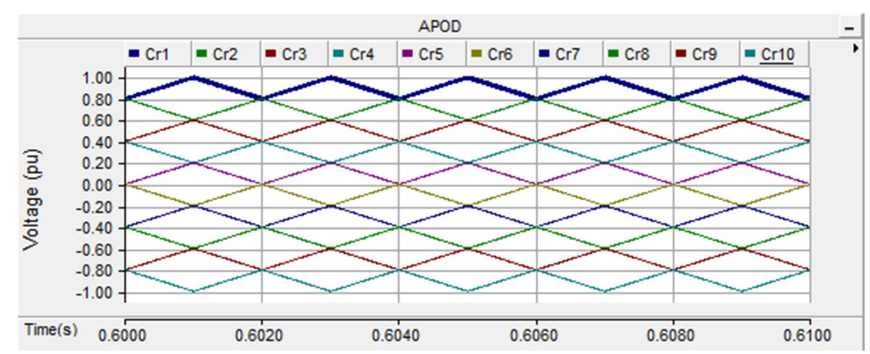

Fig. 6. Alternative phase opposite disposition PWM technique.

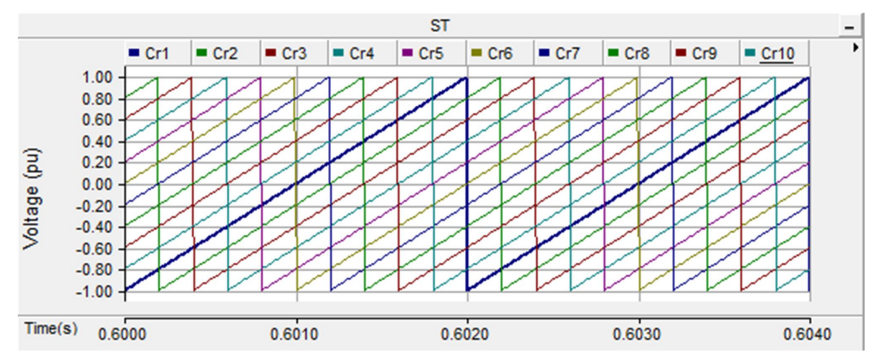

Fig. 7. Saw-tooth rotation PWM technique.

\subsubsection{Phase opposite disposition pulse width modulation}

The POD technique uses two kinds of carrier waveforms, the positive and negative carrier waveforms. The negative carrier waveforms are shifted by $180^{\circ}$ with respect to the positive carrier waveforms. The principle of this technique is shown in Fig. 5. 


\subsubsection{Alternative phase opposite disposition pulse width modulation}

In the APOD technique, the carrier waveforms are alternately displaced $180^{\circ}$ between them. The disposition of the carrier waveforms is described in Fig. 6.

\subsubsection{Saw-tooth rotation PWM technique}

The saw-tooth rotation PWM (STR-PWM) technique is similar to the PS-PWM method. The difference is that the carrier waveforms are the saw-tooth waveforms instead of the triangular waveforms as depicted in Fig. 7.

\subsection{Modulating Signal}

\subsubsection{Sinusoidal pulse width modulation}

In the SPWM technique, the modulating signal is a pure sinusoidal waveform as shown in Fig. 8. Then, this signal is compared to the carrier waveforms to generate the gating signals for the power semiconductor devices.

\subsubsection{Third harmonic injection pulse width modulation}

The THIPWM is used to improve the gain of the PWM technique. For the triangular third harmonic injection, the instantaneous value of third harmonic is calculated by

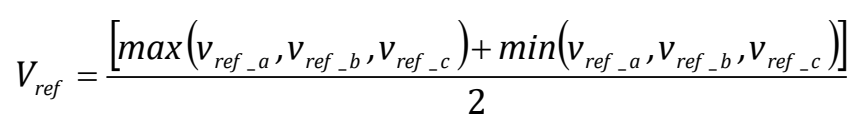

where $v_{\text {ref_a }}, v_{\text {ref_b }}, v_{\text {ref_c }}$ are phase A voltage, phase B voltage and phase $\mathrm{C}$ voltage, respectively.

The modulating voltages with THI are presented as

$$
\begin{aligned}
& v_{\text {THI_ } a}=1.1547\left(v_{\text {ref_a }}-v_{\text {ref }}\right) \\
& v_{\text {THI_b }}=1.1547\left(v_{\text {ref_b }}-v_{\text {ref }}\right) \\
& v_{\text {THI_c }}=1.1547\left(v_{\text {ref_c }}-v_{\text {ref }}\right)
\end{aligned}
$$

This modulation technique is explained in Fig. 9. The blue, green and brown curves are the sinusoidal waveform, triangular harmonic waveform and THI waveform, respectively.

\subsection{Nearest level modulation technique}

Assuming that the MMC has $N$ SMs per arm or there are $(N+1)$ levels in the output voltage. $V_{c}$ represents the average capacitor voltage of all the SMs in each phase.

$$
V_{c}=\frac{V_{d c}}{N}
$$

The number of SMs in the on-state in the upper and lower arms can be calculated by

$$
\begin{gathered}
N_{O N_{-} j u}=\operatorname{int}\left(\frac{0.5 V_{d c}-V_{r e f_{-} j}}{V_{c}}\right) \\
N_{O N_{-} j l}=\operatorname{int}\left(\frac{0.5 V_{d c}+V_{r e f_{-} j}}{V_{c}}\right)
\end{gathered}
$$

Where $j=a, b, c$. $\operatorname{int}(y)$ will get the nearest integer of $y$. At any time, total of SMs in the on-state in each phase is equal to the number of SMs per arm as given by 


$$
N=N_{O N_{-} j u}+N_{O N_{-} j l}
$$

Thus, the number of SMs in the off-state in the upper and lower arms is expressed as

$$
\begin{gathered}
N_{\mathrm{OFF}_{-} j u}=N-N_{O N_{-} j u} \\
N_{\mathrm{OFF}_{-} j l}=N-N_{O N_{-} j l}
\end{gathered}
$$

The output voltage waveform of this modulation technique is shown in Fig. 10.

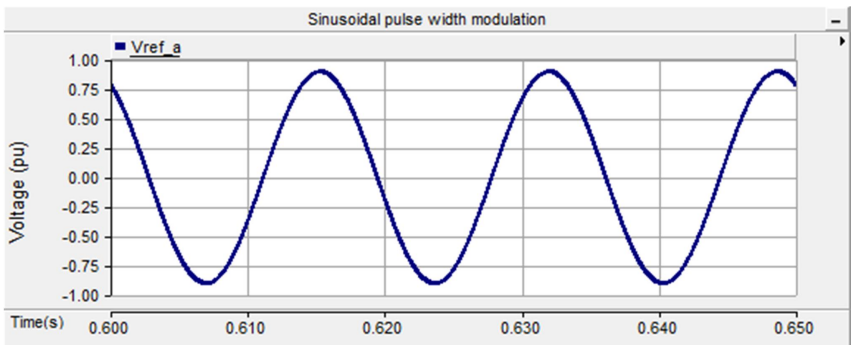

Fig. 8. Sinusoidal PWM technique.

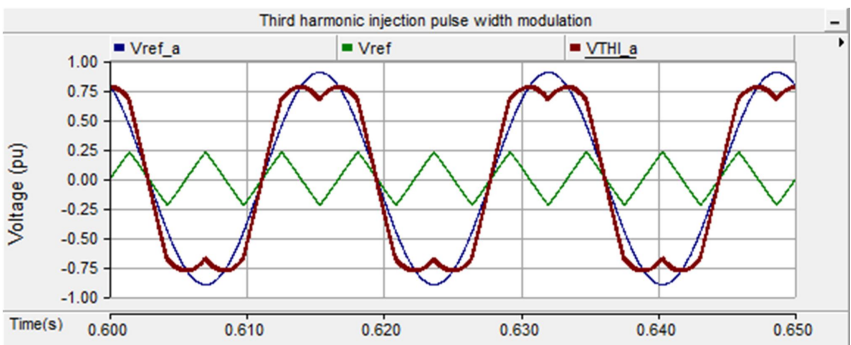

Fig. 9. Third harmonic injection PWM technique.

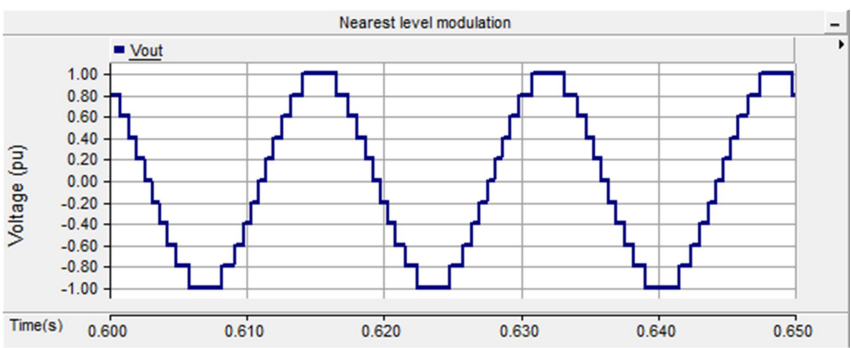

Fig. 10. Nearest level modulation technique.

\section{Simulation Results}

To analyze the modulation techniques for the MMC, several simulations have been performed by using the PSCAD/EMTDC simulation program. The parameters of the MMC are shown in Table 1. The frequency of carrier waveforms is $500 \mathrm{~Hz}$. Three modulation techniques are used in this paper, namely SPWM, THIPWM and NLM. Each modulation technique is compared to the other one based on the total harmonic distortion (THD) of the output voltage and current that is calculated as

$$
T H D(\%)=\frac{\sqrt{V_{2}^{2}+V_{3}^{2}+\ldots+V_{n}^{2}}}{V_{1}} .100(\%)
$$


Where $V_{n}$ is the RMS voltage of $n$th harmonic and $n=1$ is the fundamental frequency $(f=60 \mathrm{~Hz})$.

Table 1. Parameters of the MMC

\begin{tabular}{|l|l|}
\hline Quantity & Value \\
\hline Rated active power & $20 \mathrm{MW}$ \\
\hline Power factor & 1 \\
\hline AC voltage & $154 \mathrm{kV}$ \\
\hline Nominal frequency & $60 \mathrm{~Hz}$ \\
\hline Transformer ratio & $154 \mathrm{kV} / 33 \mathrm{kV}$ \\
\hline DC-link voltage & $\pm 60 \mathrm{kV}$ \\
\hline Number of SMs per arm & 10 \\
\hline SM capacitance & $1000 \mu \mathrm{F}$ \\
\hline
\end{tabular}

Fig. 11, Fig. 12 and Fig. 13show the output voltage waveforms corresponded to the SPWM, THIPWM and NLM techniques.

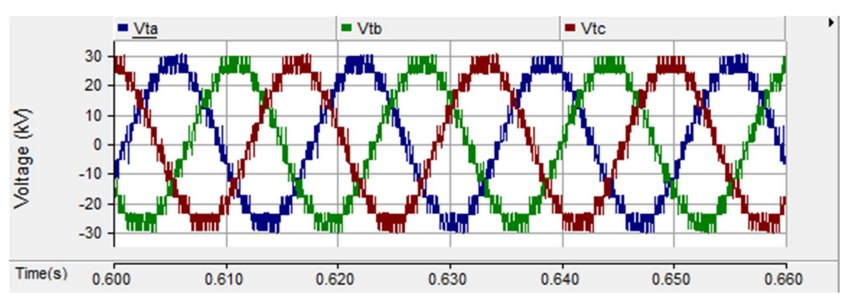

(a)

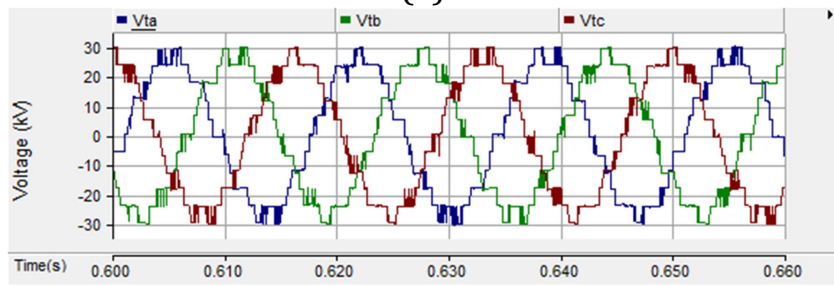

(b)

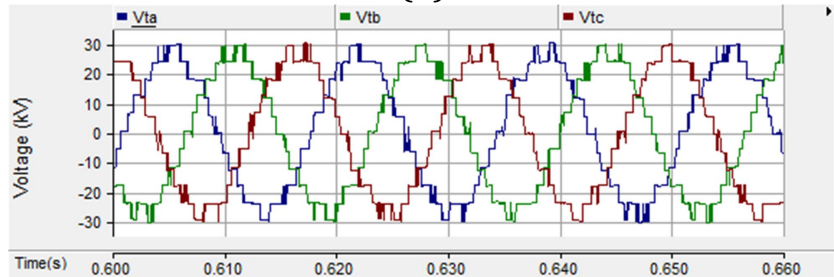

(c)

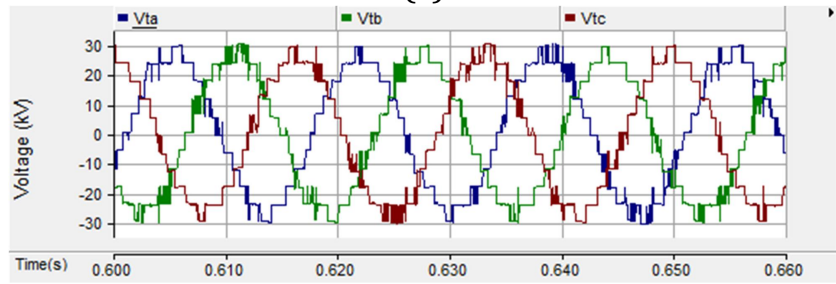

(d)

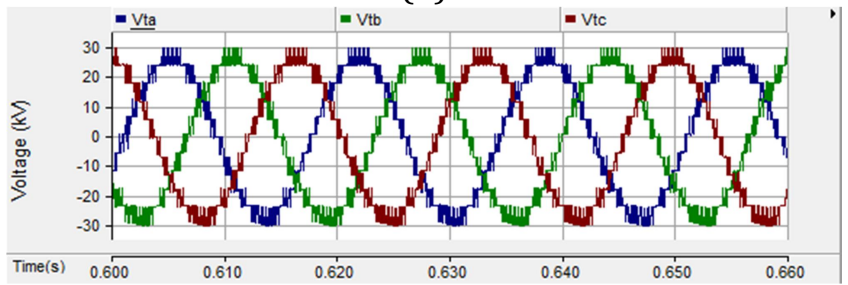

(e)

Fig. 11. Output voltage waveforms with SPWM.

(a) PS-SPWM, (b) PD-SPWM, (c) POD-SPWM, (d) APOD-SPWM, (e) STR-SPWM. 


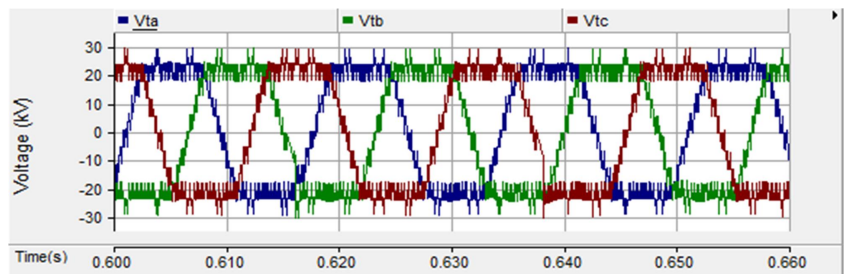

(a)

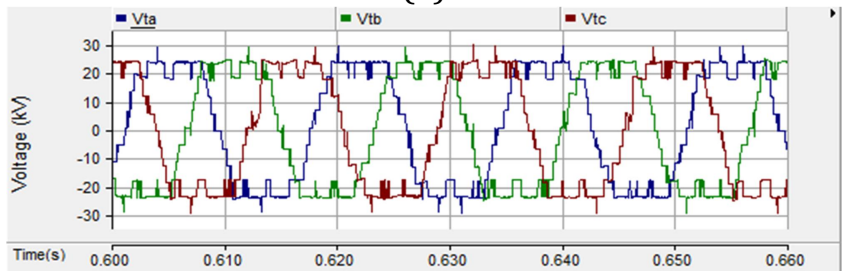

(b)

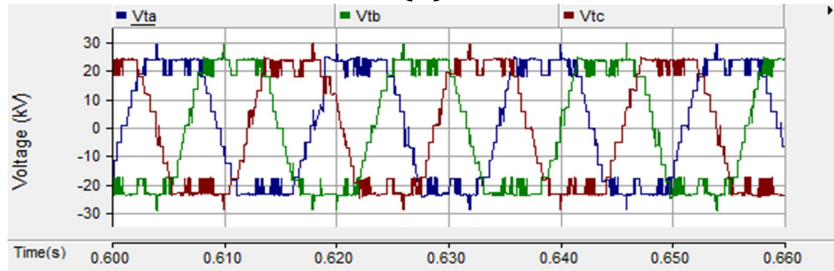

(c)

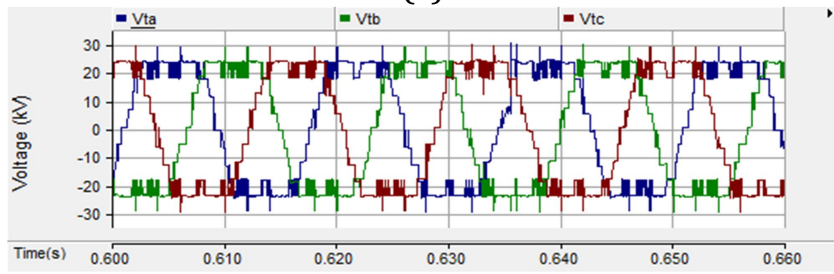

(d)

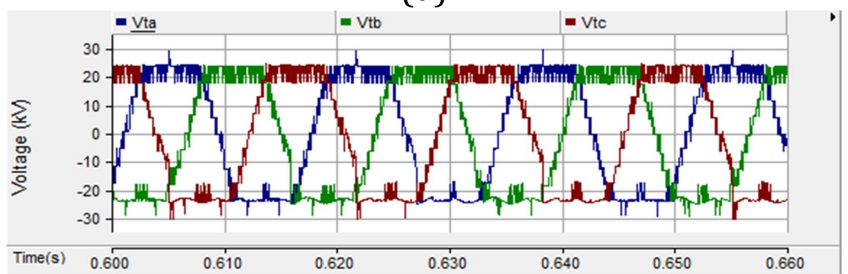

(e)

Fig. 12. Output voltage waveforms with THIPWM. (a) PS-THIPWM, (b) PD-THIPWM, (c) POD-THIPWM, (d) APOD-THIPWM, (e) STR-THIPWM

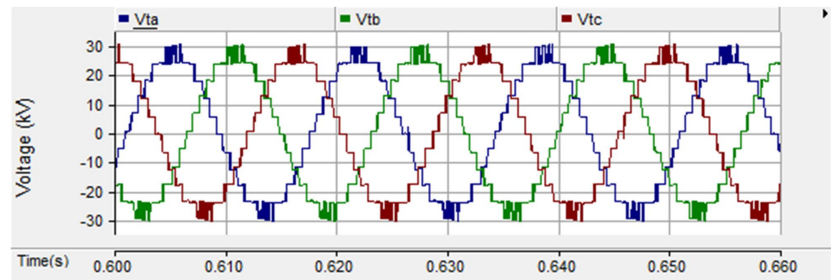

Fig. 13. Output voltage waveforms with NLM.

The PS-PWM and STR-PWM have a high switching frequency (Figs. 11(a), (e) and Figs. 12 (a), (e))because total switching frequency of all SMs in each arm is approximate $N$ times of the carrier waveform frequency, $N . f_{c r}$, while the total switching frequency is only the carrier waveform frequency, $f_{c r}$, with the PD-PWM, POD-PWM and APOD-PWM as described in Figs. 11(b), (c), (d) and Figs. 12(b), (c), (d). The NLM can achieve a lowest switching frequency because the SM is only in on-state or off-state one time per each output voltage level as shown in Fig. 13. Moreover, the NLM does not need any carrier waveforms. Therefore, it is easy to apply to the MMC. 
The THD of the output voltage and current is presented in Fig. 14 and Fig. 15, respectively. It can be seen that the THD with SPWM techniques is lower than that with the THIPWM techniques (Fig. (14)). Third harmonic injection can improve the gain of the PWM, but it increases the harmonics in the output voltage. The STR-SPWM can achieve a lowest THD (output voltage: 3.8\%, current: 1.43\%) among all modulation techniques because of the high switching frequency. The highest THD falls into the case of POD-THIPWM with the values of $23.84 \%$ for the output voltage and $9.67 \%$ for the current. The THD of the output voltage with the THIPWM techniques is almost over 20\%. The THD with the NLM keeps the third position among all modulation techniques (output voltage: 7.01\%, current: 2.02\%).

From above analysis, the PS-SPWM and STR-SPWM techniques are suitable for low level MMCs. However, these techniques can cause a high power loss. To reduce harmonics in the PD-SPWM, POD-SPWM and APOD-SPWM techniques, the level number of the MMC must be increased. The NLM technique will be a reasonable choice for high level MMCs to improve the power loss and efficiency.

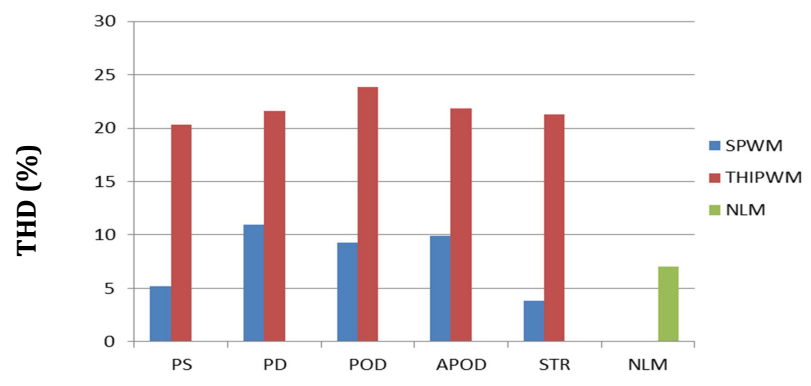

Fig. 14. THD of the output voltage.

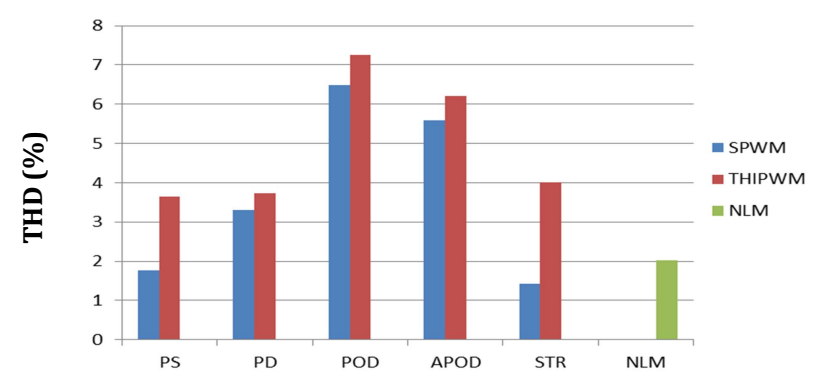

Fig. 15. THD of the current.

Besides, the relationship between the modulation index and THD is also analyzed as shown in Fig. 16 for the output voltage and Fig. 17 for the current. It can be seen that the THD of the current and the voltage with all modulation techniques is decreased when the modulation index is increased. This is due to a higher modulation index will increase the magnitude of the output voltage. Consequently, the harmonics will be lower.

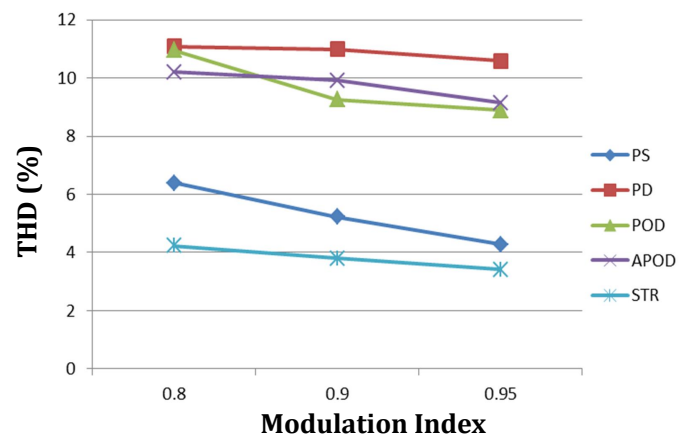

(a) 


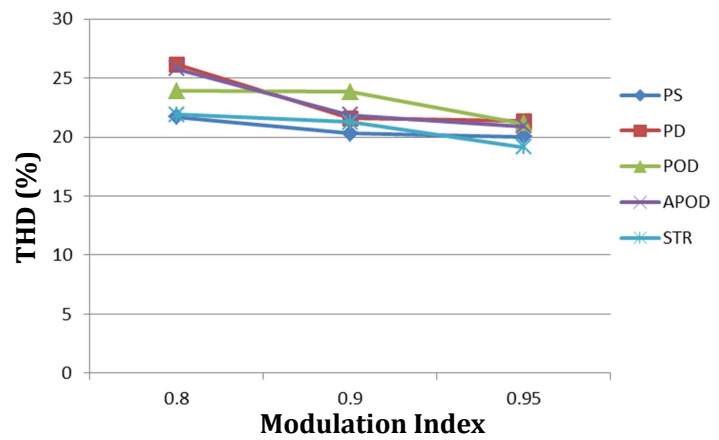

(b)

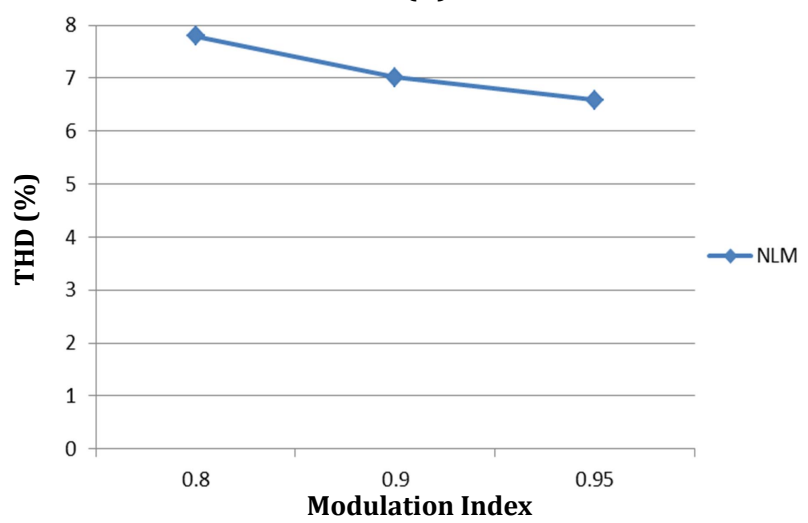

(c)

Fig. 16. The relationship between modulation index and THD of the output voltage. (a) SPWM,(b) THIPWM, (c) NLM.

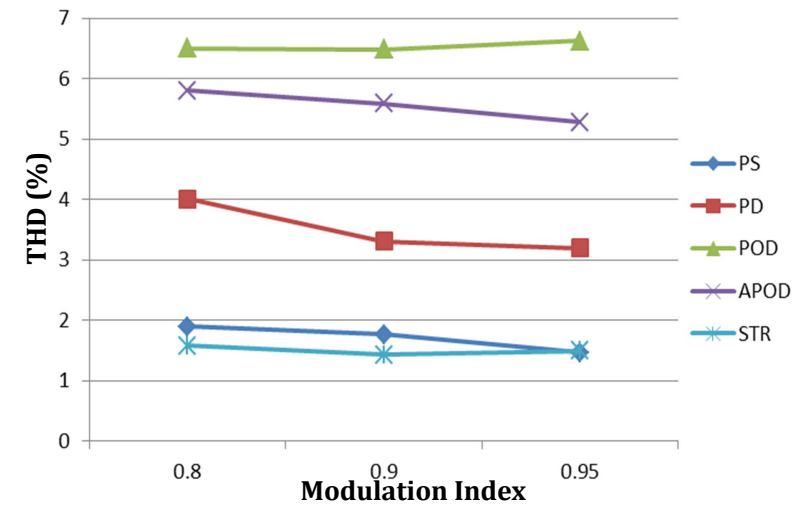

(a)

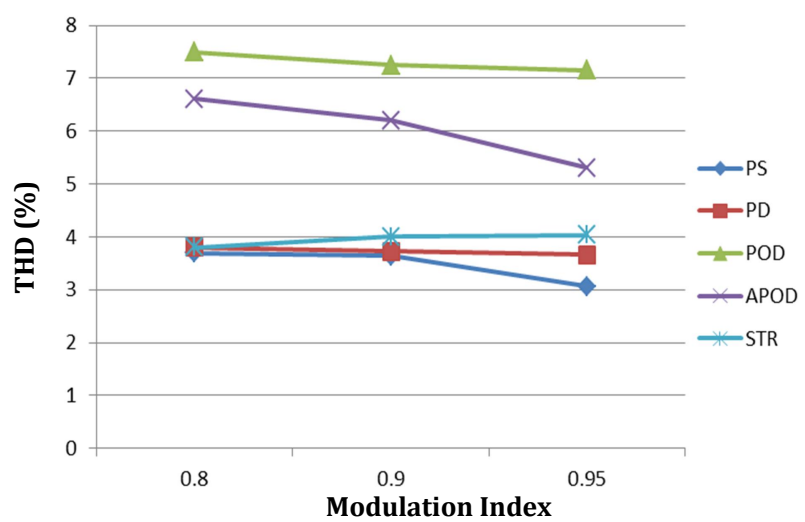

(b) 


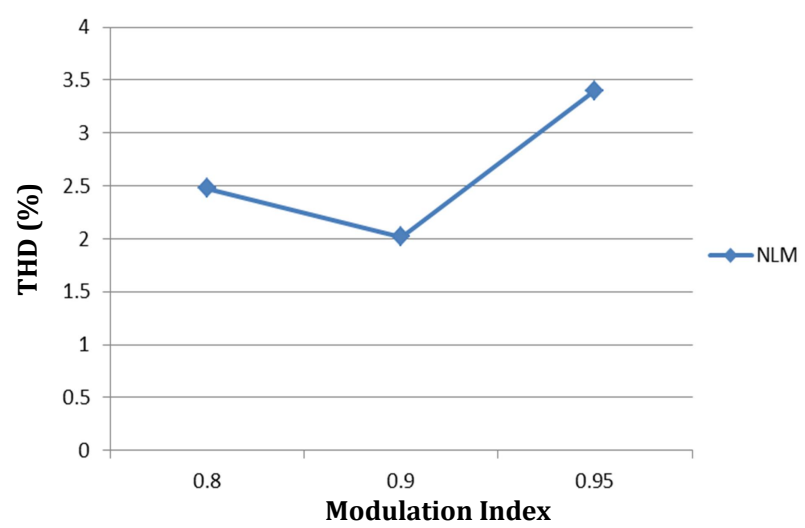

(c)

Fig. 17. The relationship between modulation index and THD of the current. (a) SPWM, (b) THIPWM, (c) NLM.

\section{Conclusion}

This paper has presented an analysis of different modulation techniques, namely SPWM, THIPWM and NLM, for the MMC. A comparison of THD between three modulation techniques has been discussed. The simulation results have demonstrated that the THIPWM techniques are not suitable for the MMC because it gives to high THD in the output voltage. The SP-SPWM and STR-SPWM techniques are only suitable for low level MMCs because it causes high power loss. The PD-SPWM, POD-SPWM, APOD-SPWM and NLM techniques are reasonable choice for high level MMCs. Especially, the NLM technique can give a lowest switching frequency among all modulation techniques. This is a necessary condition to improve the efficiency of MMC.

\section{Acknowledgment}

This work was supported by the Development of 20MWVSC HVDC for offshore wind-farm interconnection of the Korea Institute of Energy Technology Evaluation and Planning (KETEP), granted financial resource from the Ministry of Trade, Industry \& Energy, Republic of Korea. (No. 2012T100201551).

\section{References}

[1] Choi, N. S., Cho, J. G., \& Cho, G. H. (1991). A general circuit topology of multilevel inverter. Proceedings of the 22nd Annual IEEE Power Electronics Specialists Conference (pp. 96-103).

[2] Lai, J. H., \& Peng, F. Z. (1996). Multilevel converters - A new breed of power converters. IEEE Transactions on Industry Applications, 32 (3), 509-517.

[3] Tolbert, L. M., Peng, F. Z., \& Habetler, T. H. (1999). Multilevel converters for large electric drives. IEEE Transactions on Industry Applications, 35(1), 36-44.

[4] Marquardt, R., Lesnicar, A., \& Hildinger, J. (2002). Modulares stromrichterkonzept für netzkupplungsanwendungen bei hohen Spannungen. Proceedings of the ETG Conference 2002.

[5] Lesnicar, A., \& Marquardt, R. (2003). An innovative modular multilevel converter topology suitable for a wide power range. Proceedings of the IEEE Bologna Power Tech Conference.

[6] Zhao, Y., Hu, X. H., Tang, G. F., \& He, Z. Y. (2010). A study on MMC model and its current control strategies. Proceedings of the 2nd IEEE International Symposium on Power Electronics for Distributed Generation Systems (PEDG) (pp. 259-264).

[7] Allebrod, S., Hamerski, R., \& Marquardt, R. (2008). New transformer less, scalable modular multilevel 
converters for HVDC-transmission. Proceedings of the IEEE Power Electronics Specialists Conference(pp. 174-179).

[8] Saeedifard, M., \&Iravani, R. (2010). Dynamic performance of a modular multilevel back-to-back HVDC system. IEEE Transactions on Power Delivery, 25(4), 2903-2912.

[9] SirirojSirisukprasert (1999). Optimized Harmonics Stepped-Waveform for Multilevel Inverter. MSc. Thesis, Department of Electrical and Computer Engineering, Virginia Polytechnic Institute and State University (Virginia Tech).

[10] Carrara, G., Gardella, S., Marchesoni, M., Salutari, R., \& Sciutto, G. (1992). A new multilevel PWM method: A theoretical analysis. IEEE Transactions on Power Electronics, 7(3), 497-505.

[11] Baimel, D., Rabinovici, R., \& Tapuchi, S. (2014). Phase shifted PWM with third harmonic injection for over-modulation range operation. Proceedings of the international Symposium on Power Electronics, Electrical Drives, Automation and Motion (pp. 753-757).

[12] Gupta, A. K., \& Khambadkone, A. M. (2006). A space vector PWM scheme for multilevel inverters based on two-level space vector PWM.IEEE Transactions on Industrial Electronics, 53(5), 1631-1639.

[13] Guan, M., Xu, Z.,\& Chen, H. (2011). Control and modulation strategies for modular multilevel converter based HVDC system. Proceedings of the IECON 2011 - 37th Annual Conference on IEEE Industrial Electronics Society (pp. 849-854).

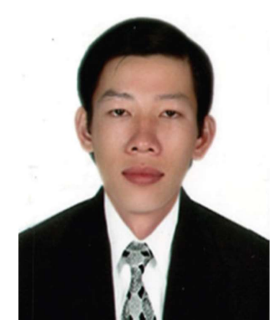

Ngoc-ThinhQuach received the B.S. degree in Electrical Engineering from Can Tho University, Vietnam, in 2007, and the M.S. and Ph.D. degrees in Electrical Engineering from Jeju National University, S. Korea, in 2012 and 2015, respectively. He is currently a Postdoctoral researcher with the Department of Electrical Engineering, Jeju National University, S. Korea. His research interests include power electronics, renewable energy systems, HVDC systems, and power system stability.

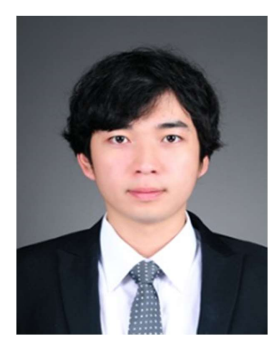

Sang HeonChae received a B.S. degree in Electrical Engineering from Jeju National University, S. Korea, in 2015. He is currently a M.S. student in Electrical Engineering, Jeju National University, S. Korea. His research interests include BESS system power electronics and microgrid.

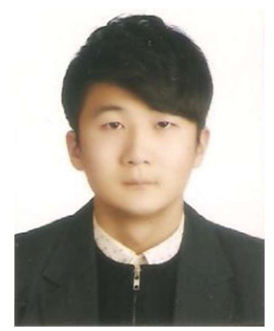

Seungmin Lee received a B.S. degree in Electrical Engineering from Jeju National University, S. Korea, in 2014. He is currently a M.S. student in the Faculty of Wind Energy Engineering Graduate School, Jeju National University, S. Korea. His research interests include wind energy systems, HVDC system, and microgrid.

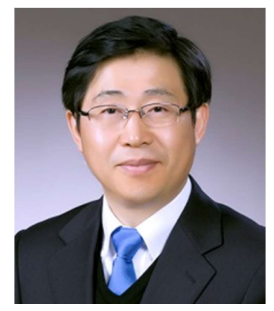

Ho-Chan Kim received his B.S., M.S., and Ph.D. degrees in Control and Instrumentation Engineering from Seoul National University in 1987, 1989, and 1994, respectively. He was a research staff member from 1994 to 1995 at the Korea Institute of Science and 
Technology (KIST). Since 1995, he has been with the Department of Electrical Engineering at Jeju National University, where he is currently a professor. He was a Visiting Scholar at the Pennsylvania State University in 1999 and 2008. His research interests include wind power control, electricity market analysis, and control theory.

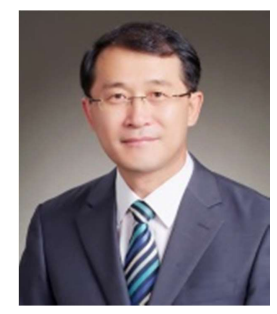

Eel-Hwan Kim received his B.S., M.S. and Ph.D. degrees in Electrical Engineering from Chung-Ang University, Seoul, Korea, in 1985, 1987 and 1991, respectively. Since 1991, he has been with the Department of Electrical Engineering, Jeju National University in Jeju, Korea, where he is currently a professor. He was a Visiting Scholar at the Ohio State University in 1995 and University of Washington in 2004. His research activities are in the area of power electronics and control, which includes the drive system, renewable energy control applications, and power quality. He is a member of KIEE, KIPE, and IEEE. 